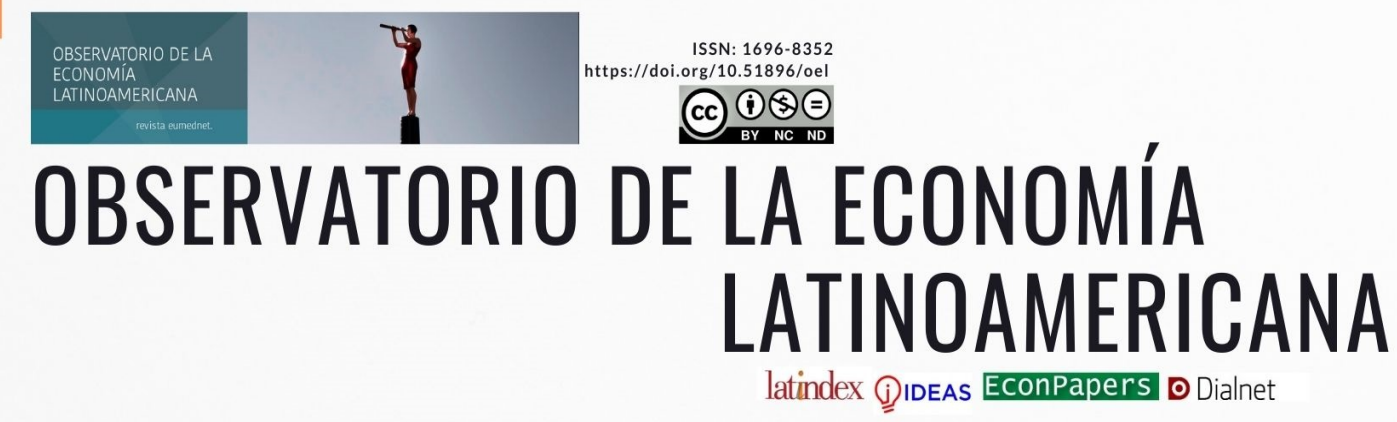

\title{
PERSPECTIVA SOCIOECONÓMICA DE LA EDUCACIÓN
}

\author{
Dra. en Educ. María del Carmen Magallanes Méndez. \\ Maestra de Tiempo Completo Plantel "Dr. Ángel Ma. Garibay Kintana" \\ de la escuela Preparatoria. \\ Universidad Autónoma del Estado de México. \\ magallanescar@yahoo.com.mx \\ mcmagallanesm@uaemex.mx \\ Mtro. en D. Werther Juárez Toledo. \\ Maestro de Tiempo Completo Facultad de Derecho. \\ Universidad Autónoma del Estado de México. \\ wjuarezt@uaemex.mx \\ Mtro. Raúl Juárez Toledo \\ Profesor de asignatura del Plantel "Cuauhtémoc" \\ de la Escuela Preparatoria. \\ Universidad Autónoma del Estado de México. \\ raultoledo85@hotmail.com
}

Para citar este artículo puede utilizar el siguiente formato:

María del Carmen Magallanes Méndez, Werther Juárez Toledo y Raúl Juárez Toledo: "Perspectiva socioeconómica de la educación", Revista Observatorio de la Economía Latinomericana (Vol 19, № 6, pp. 1-15, octubre-diciembre 2021). En línea:

https://doi.org/10.51896/oel/AGYP8412

\section{RESUMEN}

El presente ensayo muestra una perspectiva de la problemática por la que atraviesa la educación en México, desde una perspectiva socioeconómica, considerando que la educación es sin duda la mejor palanca del Desarrollo para las personas y para las distintas naciones, sin embargo, existen factores de índole socioeconómico, que han puesto límites al desarrollo de la educación en México, por lo que es necesario, replantear el quehacer de los gobiernos, los ciudadanos y la cooperación internacional, si se quiere que los niveles educativos aumenten y con ello mejorar la calidad de vida de la población. Se debe considerar que en México, las limitaciones históricas han incidido directamente para que la educación no alcanzará los niveles de desarrollo deseado, no obstante de que en la última década (2010 - 2020) y ante exigencias internacionales, se han tenido avances en cobertura, inversión pública y resultados de evaluaciones, sin embargo, siguen siendo insuficientes, por lo anterior es necesario cuestionarse ¿por qué el país no crece en materia educativa en comparación con otras regiones del mundo? y por otro lado, ¿de qué depende que los países sean tan desiguales en la materia? El presente estudio arroja una arista de la problemática por la que atraviesa la educación en México, con base en fenómenos como: pobreza y desigualdad. Con 
lo que se pretende tratar de entender los retos que hay que superar para alcanzar el tan anhelado desarrollo.

Palabras claves: Educación, economía, pobreza, ingresos y desigualdad.

\section{SOCIOECONOMIC APPROACH OF THE EDUCATION}

\section{ABSTRACT}

From a socioeconomic perspective, the present study shows some of the problems Mexican education faces, considering that education is the best development lever for people and nations. However, socioeconomic factors have limited the development of education in Mexico, so governments, citizens, and international cooperation should be rethink if educational levels are to increase and improve the quality of education. In Mexico, historical limitations have had a direct impact in limiting the development levels of education, even though in the last decade (2010 - 2020) and response to international demands, there have been advances in coverage, public investment, and evaluation results. Therefore, it is necessary to question why Mexican education does not grow compared to other regions of the world? And on the other hand, where does this inequality among countries come from? This study reviews the problem that Mexican education is going through, focusing on poverty and inequality. The aim is to understand the challenges that must be overcome to achieve the longawaited development.

Keywords: Education, economy, poverty, income and inequality.

\section{INTRODUCCIÓN}

La educación en México mantiene relación directa con fenómenos históricos característicos del país, por un lado, las distintas etapas de formación política, posteriores a la colonia, marcaron las directrices de la creación de las instituciones educativas, con líneas ideológicas particulares, e influencia de distintas regiones del mundo, con apego al mundo Occidental, pero sin olvidar del todo el multicultural pasado prehispánico. La región, es resultado del mestizaje desigual, que mantuvo un esquema de castas con privilegios en orden piramidal, lo que llevó a generar una tradición revolucionaria, gestada desde el siglo XIX y que continúa hoy en día, en el ideario político. El sueño de la integración económica de las distintas clases sociales del México revolucionario se fue diluyendo ante los desiguales resultados de los distintos modelos, que arrojaron grandes problemáticas económicas agudizadas por las constantes crisis y devaluaciones. Lo que ha desembocado en una sociedad de aproximadamente 127 millones de múltiples realidades y una historia en común, diversidad de culturas que convergen en un mundo que gira entre el pasado nostálgico prehispánico y el presente convulso por su proceso histórico, mundos milenarios y sociedades modernas, megalópolis y provincias despobladas, llena de recursos naturales, pobre y rica a la vez; así vive día a día la gente en el sureste, centro y norte mexicano. El espacio geográfico mexicano de gran pluriculturalidad y a su vez un país con muchas carencias que detonan una gran cantidad de fenómenos negativos que se han convertido en verdaderos obstáculos a la vida. 
Por otro lado, el desarrollo económico del país ha sido heterogéneo, diverso y centrado en un esquema de clases privilegiadas que mantienen férreo control de la economía, haciéndose evidente la desigualdad en las diversas regiones del país. Como consecuencia México tiene indicadores socioeconómicos que permiten iniciar un debate de su injerencia sobre los limitados resultados educativos, y en sinergia, las condiciones de carencia de gran parte de la población mexicana a consecuencia de los bajos indicadores educativos, que limitan su inserción a los beneficios de la economía capitalista. Para efectos del presente ensayo consideraremos dos principales fenómenos: la pobreza y la desigualdad, en función de considerar que su atención debe de ser prioritaria para poder tener los mínimos de bienestar y con ello las posibilidades de competir en el mundo globalizado que exige cada vez más estándares elevados y capacidades especializadas, y por otro lado, considerando que la educación es y será la mejor palanca del desarrollo personal y por ende de las distintas naciones, es de reconocerse, que no se podrán superar los grandes problemas de un país sin considerar a la educación como la base del desarrollo nacional, que se traduce en términos de John Rawls en “Libertad y Justicia Social” (citado en Osorio García, 2012, p. 151).

Con base en ello, se considera impostergable que el eje central en la agenda pública mexicana sea la "Educación de Calidad", indicada en el Objetivo 4 de la Agenda 2030 para el Desarrollo Sostenible, en función de considerar que el ODS 4, constituye la síntesis de las ambiciones de la educación, cuyo objetivo es "garantizar una educación inclusiva, equitativa y de calidad y promover las oportunidades de aprendizaje durante toda la vida para todos" de aquí al 2030; mismo que coloca el tema como la base para mejorar la vida, considerando que no puede haber libertad si no hay entendimiento de la vida y capacidades para potencializar y elegir el futuro, por lo que propone alcanzar la universalidad, por lo menos en la educación básica, impulsar la tecnificación de la educación para el trabajo, el aumento de la inversión pública, la disminución de la brecha de género y el trabajo cooperativo entre las naciones.

Por ello, se desarrolla el presente ensayo, que tiene como propósito dar una visión socioeconómica, de las grandes problemáticas por las que atraviesa la educación en México. Con lo que se pretende tratar de entender, por un lado, los retos que hay que superar para alcanzar el tan anhelado desarrollo y por otro la correlación que existe entre educación y la economía, que para este caso se considera como una histórica limitante para un gran sector de la población mexicana.

La investigación documental se basa en el método de análisis comparativo, con el uso de fuentes oficiales tanto nacionales como internacionales, entre las que destacan: la Organización de las Naciones Unidas (ONU), La Organización para la Cooperación y el Desarrollo Económicos (OCDE), La Comisión Económica para América Latina y el Caribe (CEPAL), el Banco Interamericano de Desarrollo (BID), los estudios realizados por la organización internacional "OXFAM", el Instituto Nacional de Estadística, Geografía e Informática (INEGI), El Consejo Nacional de Evaluación de la Política de Desarrollo Social (CONEVAL) entre otras. Para el caso de la pobreza, es necesario considerar que se analizará desde una óptica de Necesidades y Carencias de 
satisfactores básicos, considerando que existen líneas de bienestar que nos permitirán conocer el contexto mexicano de manera objetiva.

\section{DESIGUALDAD Y POBREZA EN MÉXICO}

La desigualdad, es uno de los mayores problemas a los que se enfrenta la población mexicana, destacando que el capital de los mexicanos más ricos en 2017 fue de 116 mil millones de dólares, esto significa que las 10 personas más ricas de México acumulan la misma riqueza que el $50 \%$ más pobre del país (Fuentes Nieva y Barrón, 2018). En el documento Human Development Report 2020 del Programa de las Naciones Unidad (PNUD), se indica que México para 2019 alcanzó un Índice de Desarrollo Humano (IDH) de 0.779 (IDH Alto), posicionándose en el lugar 74 de 189 países, lo que debería de entenderse como una población con características de vida estables, con acceso a salud, educación e ingreso suficiente para mantener una vida digna, sin embargo, cuando se descuenta el valor de la desigualdad, el IDH cae a 0.613 conforme al Índice de Desarrollo Humano Ajustado por Desigualdad (IHDI), teniendo una pérdida general de $21.3 \%$ debido a la desigualdad en la distribución de los índices de las dimensiones del IDH, de los cuales el de mayor pérdida es el de ingresos, el cual cae un 33.4\%, seguido del coeficiente de educación el cual cae un $18.4 \%$, y en tercer lugar el coeficiente de salud (expectativa de vida) que cae un $10.5 \%$ (ver tabla 1 ).

\section{Tabla 1.}

México: Índice de Desarrollo Humano Ajustado (IHDI) 2019

\begin{tabular}{|c|c|c|c|c|c|c|}
\hline \multirow[b]{2}{*}{ País } & \multicolumn{6}{|c|}{2019} \\
\hline & $\begin{array}{c}\text { Valor IHDI } \\
\text { (\%) }\end{array}$ & $\begin{array}{c}\text { Pérdida } \\
\text { general } \\
(\%)\end{array}$ & $\begin{array}{c}\text { Coeficiente } \\
\text { de } \\
\text { desigualdad } \\
\text { humana } \\
\text { (\%) }\end{array}$ & $\begin{array}{c}\text { Desigualdad } \\
\text { en la vida } \\
\text { expectativa } \\
\text { en } \\
\text { nacimiento } \\
\text { (\%) }\end{array}$ & $\begin{array}{c}\text { Pérdida } \\
\text { debido a la } \\
\text { desigualdad } \\
\text { en } \\
\text { educación } \\
(\%)\end{array}$ & $\begin{array}{c}\text { Pérdida } \\
\text { debido a la } \\
\text { desigualdad } \\
\text { en los } \\
\text { ingresos } \\
(\%)\end{array}$ \\
\hline México & 0.613 & 21.3 & 20.8 & 10.5 & 18.4 & 33.4 \\
\hline
\end{tabular}

Nota: Datos tomados de Human Development Report. México, 2020. UNDP.

Lo pasado se puede considerar como una situación álgida para la sociedad mexicana, debido a que impacta negativamente en los hogares menos favorecidos económicamente, en aspectos tales como "el educativo", muestra de ello se aprecia en la tabla 2, donde se nota una clara desventaja entre el promedio de años de estudio del en hogares que viven "bajo la línea de pobreza extrema" y aquellos que viven en hogares "bajo la línea de pobreza". El clima educacional entre los jefes de familia (jefe de hogar y su cónyuge) que viven en un hogar "bajo la línea de la pobreza" tiene más de probabilidades de estudiar que aquellos que se encuentran en "pobreza extrema. Advertimos que en los hogares que viven "bajo la línea de la pobreza" un 39\% más de jefes de familia cuentan con al menos primaria completa, $24.4 \%$ más que cursaron la secundaria y un $3.3 \%$ más cuentan con 
estudios superiores, en comparación con los jefes de familia que viven en hogares cuyo estatus es "la pobreza extrema".

\section{Tabla 2.}

México. Incidencia de la pobreza y la pobreza extrema según clima educacional del hogar, total 2018 (porcentajes)

\begin{tabular}{|c|c|c|c|c|c|c|c|c|c|}
\hline \multicolumn{4}{|c|}{ Población bajo la línea de pobreza extrema según: } & \multicolumn{5}{c|}{ Población bajo la línea de pobreza según: } \\
\hline \multicolumn{4}{|c|}{ Clima educacional del hogar: a/ } \\
\hline $\begin{array}{c}\text { Primaria } \\
\text { incompleta }\end{array}$ & $\begin{array}{c}\text { Secundaria } \\
\text { incompleta }\end{array}$ & $\begin{array}{c}\text { Secundaria } \\
\text { completa }\end{array}$ & $\begin{array}{c}\text { Terciaria } \\
\text { incompleta } \\
\text { (técnica) }\end{array}$ & $\begin{array}{c}\text { Terciaria } \\
\text { completa }\end{array}$ & $\begin{array}{c}\text { Primaria } \\
\text { incompleta }\end{array}$ & $\begin{array}{c}\text { Secundaria } \\
\text { incompleta }\end{array}$ & $\begin{array}{c}\text { Secundaria } \\
\text { completa }\end{array}$ & $\begin{array}{c}\text { Terciaria } \\
\text { incompleta } \\
\text { (técnica) }\end{array}$ & $\begin{array}{c}\text { Terciaria } \\
\text { completa }\end{array}$ \\
\hline 21.4 & 9.9 & 3.4 & 1.5 & 0.4 & 60.4 & 45.4 & 27.8 & 12.2 & 3.7 \\
\hline
\end{tabular}

Nota: Datos tomados de la Comisión Económica para América Latina y el Caribe (CEPAL), sobre la base de Banco de Datos de Encuestas de Hogares (BADEHOG).

Clima educacional del hogar se refiere al promedio de años de estudio del jefe de hogar y su cónyuge. En el caso en que no hubiera cónyuge del jefe en el hogar, corresponde a los años de estudio del jefe.

La desigualdad económica da origen a limitaciones en cuanto a la educación, por tres principales factores:

A.- La falta de recursos económicos, que impiden la cobertura de las necesidades mínimas para la asistencia a la escuela, tan grave puede ser la situación que no cuenten con los mínimos nutricionales para poder mantener la propia vida;

B.- La falta de recursos económicos en un grupo familiar obliga a los miembros de la familia migrar, insertarse en el ámbito laboral a temprana edad, en muchos casos en ambientes no formales y/o ilegales;

C.- El fenómeno de la marginación puede excluir a las personas de los servicios públicos escolares.

Por lo pasado es necesario dirigir esfuerzos por considerar que al hablar de educación, indudablemente es necesario hablar de economía, de ahí que erradicación de la pobreza sea el primer Objetivo de Desarrollo Sostenible de la Agenda 2030, ya que es una condición que trastoca prácticamente todos los esquemas humanos, impidiendo potencializar las capacidades y delimitando el entendimiento y con ello la libertad, considerando que va más allá de la falta de un empleo o un ingreso económico. La pobreza, es una realidad en la que se encuentra sumida una gran parte de la población mexicana, siendo que para el año 2018 el Consejo Nacional de Evaluación de la Política de Desarrollo Social (CONEVAL), informa que en México un total de 52.4 millones de personas se encontraban en alguna situación de pobreza, lo que equivalía al $41.9 \%$ del total de la población, de estos 34.5\% (43.1 millones) se encontraban en situación de "pobreza moderada" (aquellas personas que, siendo pobre, no son pobre extremos). Por otro lado, el $7.4 \%$ (9.3 millones de personas) se 
encontraban en una condición de "pobreza extrema" (cuando se tiene tres o más carencias, de seis posibles, dentro del Índice de Privación Social y que, además, se encuentra por debajo de la línea de bienestar mínimo). Las personas en esta situación disponen de un ingreso tan bajo que, aun si lo dedicase por completo a la adquisición de alimentos, no podría adquirir los nutrientes necesarios para tener una vida sana).

En contraste, según el propio CONEVAL (2018) el 21.9\% (27.4 millones) de la población no era considerado como pobre ni vulnerable (aquella población cuyo ingreso es superior a la línea de bienestar y que no tiene ninguna de las carencias sociales que se utilizan en la medición de la pobreza). Lo que hace a México un país polarizado (muchos pobres y pocos ricos) posicionado entre los 10 países más desiguales del mundo. En la gráfica 1 se aprecia que el 48.8\% (61.1 millones de mexicanos) percibe un ingreso inferior a la línea de pobreza y un $16.8 \%$ (21.1 millones de compatriotas) tiene un ingreso inferior a la línea de pobreza extrema.

\section{Fotografías. México: Pobreza rural}
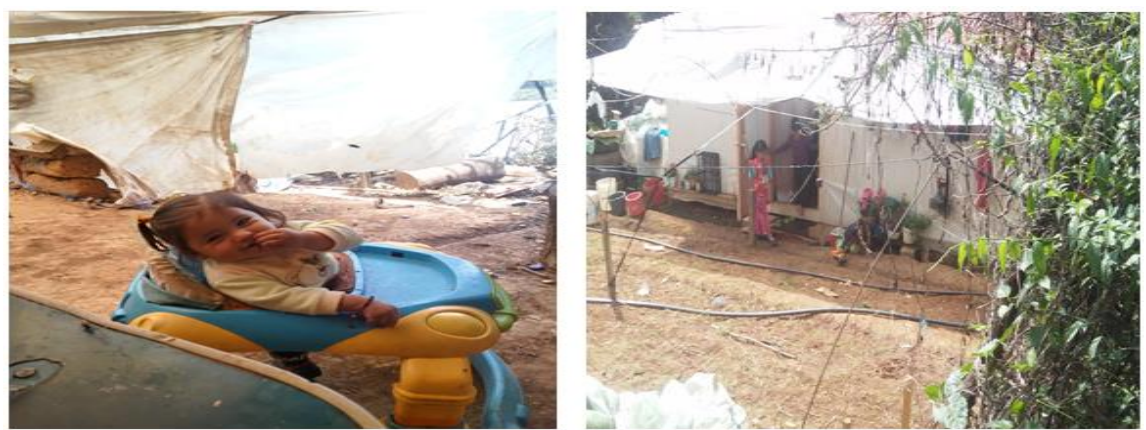

Fuente: Fuente: José Antonio Orozco Magallanes, 2020.

El 72.0\% de la población mexicana vive en hogares cuyos ingresos per cápita son inferiores a 1.8 líneas de pobreza, por lo que son considerados como "Estratos bajos"; el 36.1\% en "Estratos medios" y únicamente el $1.1 \%$ pertenece a "Estratos altos". Valor que la CEPAL (2019) considera mínimo para bajar el riesgo de caer en una situación de pobreza ante episodios coyunturales como la pérdida del empleo. Esta realidad que se forjó por más de 5 décadas y que ha provocado que la brecha entre pobres y ricos mexicanos se haga cada vez más amplia generando entre la población mexicana, menos favorecida económicamente, entre otras situaciones:

$>$ Rezago educativo (16.9\%)

$>$ Acceso a los servicios de salud (16.2\%)

$>$ Acceso a la seguridad social $(71.7 \%)$

$>$ Acceso a la alimentación (20.4\%)

$>$ Calidad y espacios de la vivienda (11.1\%) y

$>$ Acceso a los servicios básico en la vivienda (19.8\%). 


\subsection{Desigualdad educativa}

De acuerdo con el Banco Mundial "La educación es un derecho humano, un importante motor del desarrollo y uno de los instrumentos más eficaces para reducir la pobreza y mejorar la salud, y lograr la igualdad de género, la paz y la estabilidad. Además de generar beneficios elevados y constantes en términos de ingreso, constituye el factor más importante para garantizar la igualdad de oportunidades".

México está muy lejos de alcanzar lo expuesto por el Banco Mundial, esto basado en el documento denominado ¿Cómo va la vida en México? publicado por la OCDE en el año 2017 en el que entre otras situaciones indica, que el país en la Dimensión "Educación y habilidades" en el indicador "Logro educativo" se tiene un $64.48 \%$ en proporción de adultos de 25 a 64 años con educación básica, por arriba del promedio de la OCDE que es de $22.83 \%$; para el rubro de "Habilidades del estudiante" encontramos que el 33.78\% de estudiantes de 15 años obtuvieron un puntaje igual o inferior al "Nivel 2" en Ciencias, Lectura y Matemáticas en prueba PISA, $20.82 \%$ más que el promedio de países de la OCDE.

Hay que considerar que para la educación Media Superior y Superior la brecha de la desigualdad se encuentra abismalmente ensanchada en comparación con el acceso a los niveles de la educación primaria, en 2018 únicamente el 18\% de la población mexicana de 25 a 64 años contaba con estudios superiores, muy por debajo de la media de los países de la OCDE que es de $38.6 \%$, similar situación se presenta para las personas que cuentan con estudios de nivel medio superior, en el país sólo el $21.2 \%$ cuenta con ello, $22.8 \%$ menor al promedio de la OCDE.

En México, tan solo el $0.05 \%$ de los que obtengan un título universitario estudiarán un posgrado, esto tendrá impacto en el tipo de mano de obra y fuerza productiva con la que cuenta y contará el país, sin considerar el tipo de cosmovisión de vida. Otro tipo de desigualdad que se presenta en México es el acceso tecnológico, que generan divergencias en la comunicación, en las nuevas herramientas de la educación y los recursos de la tecnología de la información y crea un fenómeno excluyente entre las necesidades del mercado laboral y las competencias necesarias. La llamada segregación digital, que pone de manifiesto la diferencia entre los que tiene accesos a la nueva era tecnológica y los que por cuestiones de infraestructura, economía o conocimiento no han logrado ingresar.

En México, la segregación digital se hace presente ante la falta de infraestructura que permita el uso cotidiano de las Tecnologías de la Información y Comunicación, en algunos casos los recursos son limitados, considerando que la tecnología requiere no solo su compra, sino que también su mantenimiento y actualización constante. Por otro lado, se debe considerar las competencias del docente ante una brecha generacional que impone un mundo tecnológico cambiante aunado a la inversión que se necesita para capacitar en el uso de las TIC's. En tema es necesario considerar que las nuevas vertientes educativas se basarán en el uso de las TIC's, sin embargo, sería necesario 
revisar si la infraestructura educativa y la prospectiva de inversión, sobre todo del nivel básico, se encuentra acorde con el devenir educativo.

En la era posmoderna, la tecnología marca una de las brechas de desigualdad que tendrá gran relevancia en un futuro, puede ser tan importante que determine las condiciones laborales y por ende de vida de los habitantes. Sin duda es un gran reto para el país.

La "Educación Pública Mexicana" como producto social e inmerso en el ámbito de la sociedad cognitiva, tiene varios retos para responderle a la sociedad entre ellas:

> Implementar políticas que impulsen el desarrollo de la ciencia, la tecnología y la innovación factibles y ajustadas a sus recursos institucionales, lo anterior bajo un tenor nacional de crisis económica endémica;

> Invertir en infraestructura educativa que responda a las nuevas necesidades tecnológicas;

> Elevar la calidad de la educación impartida en todos los niveles y;

> Vincular a la educación media superior y superior con el ámbito laboral, no solo como respuesta al mundo globalizado, sino con una visión humanística y ética.

\section{DESIGUALDAD, EDUCACIÓN Y MERCADO LABORAL}

El Objetivo 8 de la Agenda 2030 referente "promover el crecimiento económico inclusivo y sostenible, el empleo y el trabajo decente para todos.", se vincula con la idea de que es la educación la forma efectiva que permite la movilidad socioeconómica ascendente y es clave para salir de la pobreza". Al mismo tiempo señala que un crecimiento económico inclusivo y sostenido puede impulsar el progreso, crear empleos decentes para todos y mejorar los estándares de vida. Con base en lo anterior, es innegable que en el actual ámbito de globalización es cada vez más firme la convicción de que la educación y las capacidades científicas constituyen la principal arma competitiva de las economías nacionales, idea que parte de una estrecha relación entre la capacitación laboral, la difusión de nuevas tecnologías y el proceso de innovación. Situación que se vislumbra difícil para predecir un ascenso social de la población mexicana, debido a que son décadas de descuido al no generar políticas públicas que apuntaran a una vida sostenible en cada hogar mexicano, que ha derivado en la imposibilidad de alcanzar lo mínimo indispensable para aspirar a una digna "calidad de vida". Refiriéndonos a la percepción laboral, se destaca que entre más grados de estudio posea una persona, mayor es su percepción laboral, situación que el INEGI (2018) da a conocer al indicar que una persona con estudios de "a lo más primaria completa" gana aproximadamente 2,842 pesos al mes (más o menos 95 pesos al día); contrario a ello los que cursaron un "Posgrado sea completo o incompleto" percibe 28,960 pesos al mes (alrededor de 965 pesos por día). Situación muy desigual, que ensancha cada vez más la brecha entre uno y otro sujeto. 


\section{Tabla 3.}

México: Ingreso promedio trimestral monetario por nivel de escolaridad 2018

\begin{tabular}{|l|l|}
\hline \multirow{2}{*}{ Nivel de escolaridad } & $\begin{array}{l}\text { Ingreso } \\
\text { (pesos) }\end{array}$ \\
\cline { 2 - 2 } & Total \\
\hline A lo más primaria completa & 8527 \\
\hline Secundaria completa o incompleta & 13846 \\
\hline Preparatoria completa o incompleta & 19316 \\
\hline Profesional completa o incompleta & 37177 \\
\hline Posgrado completo o incompleto & 86880 \\
\hline
\end{tabular}

Fuente: Elaboración propia con datos obtenidos de la ENIGH 2018.

La desigualdad de ingresos, considerando el grado máximo de estudios de los mexicanos la podemos distinguir en la gráfica 2, en la misma advertimos que la concentración de la Población Económicamente Activa (PEA) que tiene una percepción menor a 1 salario mínimo (SM) que equivale aproximadamente a 4.5 dólares, hasta aquellos que perciben 5 salarios y más, igual se aprecia la población que a pesar de desempeñar una labor ocupacional no percibe salario alguno. Muy poca población mexicana recibe 5 salarios mínimos, en este rubro tenemos un $7.0 \%$ de la población que cuenta con primaria, $14.0 \%$ con estudios de secundaria y el $79.0 \%$ que cuentan con Nivel Medio Superior. Pero se distingue que la PEA que cuenta con estudios de NMS disminuyó en un $27.8 \%$ en 3 años, al pasar de 2, 417, 691 individuos en 2015 a 1, 747, 014 personas en 2018.

\section{Gráfica 2.}

Población Económicamente Activa, 2015 y 2018 (por número de salarios mínimos, educación y primer trimestre)

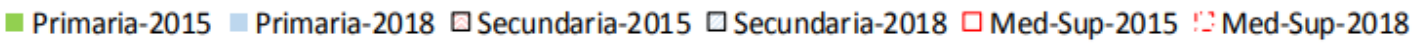

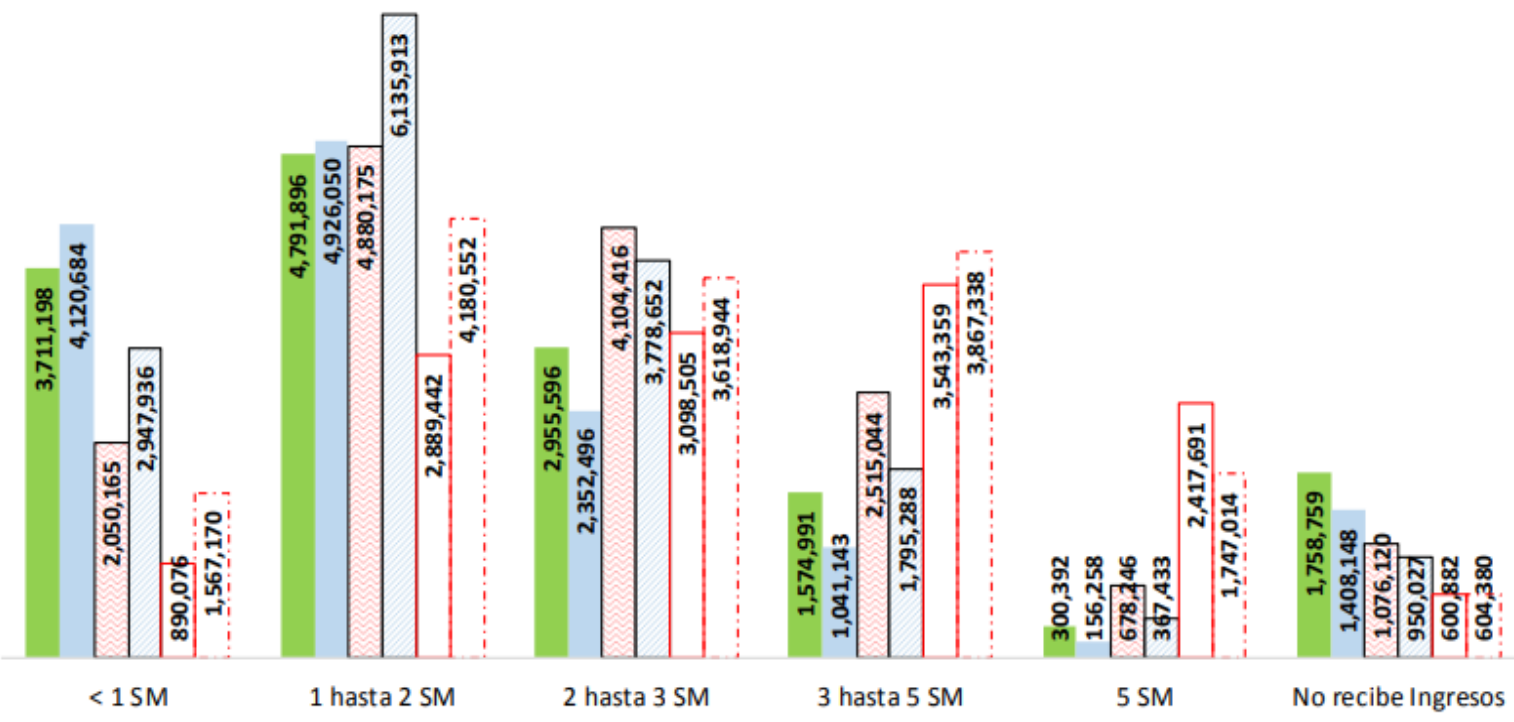


Fuente: Gráfica obtenida de Caracterización del Mercado Laboral en México. CEFP.

\subsection{Educación trabajo formal e informal}

En la gráfica 3 se muestra que en México en el 2018, entre más preparación educativa poseía un sujeto mayor es su posibilidad de insertarse a laborar en el sector "formal". De igual forma se distingue que el $42.8 \%$ trabajadores que poseen estudios superiores se inserta en el sector formal, en contraste del $10.1 \%$ que labora en el sector informal. Desde el punto de vista de la ocupación, según el INEGI (2019) el concepto de informalidad comprende distintas modalidades que van desde el típico micronegocio urbano no registrado ante la autoridad tributaria (Sector Informal) hasta formas de trabajo utilizadas por unidades económicas formales que no les dan acceso a la seguridad social.

En cuanto al trabajo formal e informal de personas que cuentan con estudios de educación básica encontramos que el $28.7 \%$ y el $46.8 \%$ de la población con estudios de nivel primaria y secundaria (respectivamente) se dediquen al trabajo informal, y un $9.7 \%$ y el $41.9 \%$ que cuentan con estudios de nivel primaria y secundaria (respectivamente) se desempañan en el trabajo formal.

\section{Gráfica 3.}

México. Distribución de trabajadores según nivel de educación

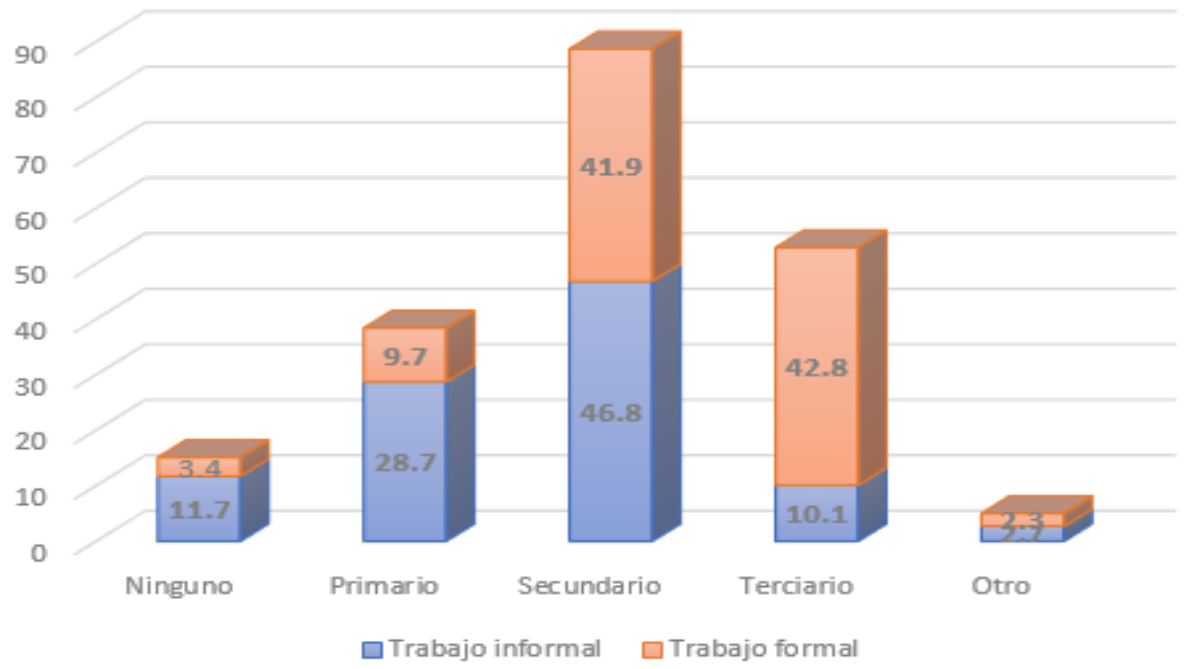

Fuente: Elaboración propia con datos obtenidos de la OIT 2018.

De acuerdo con el PNUD (2020), la desigualdad de ingreso a su vez se correlaciona con la movilidad social, condicionando los logros de las personas en mayor medida a sus orígenes y en menor medida a sus propios méritos. En México al menos 7 de cada 10 personas que nacen en el quintil más pobre no logran superar la línea de pobreza, situación que se agudiza en la región sur del país. Los datos identifican como elementos determinantes de la movilidad en México a aquellos relacionados con la riqueza del hogar de origen, la región de nacimiento, el logro educativo de los padres, el tono de piel, las condiciones de la localidad de origen, el sexo de la persona y si son 
padres y madres hablantes de lenguas indígenas. La baja movilidad se concentra en los extremos de la distribución socioeconómica, de manera que quienes nacen en posiciones de mayor desventaja poseen pocas posibilidades de superar su situación, y quienes se encuentran en una posición privilegiada tienen reducidas probabilidades de perder su estatus.

Para el caso de la ocupación en México, la OCDE (2018) indica que "un nivel de educación alto está correlacionado con niveles bajos de desempleo, por tanto, a menor nivel educativo mayor es la probabilidad de estar desempleado". Afirmación que no se da en México. En el ámbito laboral, en México, pasa lo que no pasa en ningún otro país miembro de la OCDE (2018), la tasa más alta de desempleo se da entre los más educados $6 \%$, contra $3 \%$ de tasa de desocupación entre personas con educación inferior a la secundaria. En el país, considerando información de la Secretaría del Trabajo y Previsión Social (2019), tener nivel alto de instrucción no garantiza tener empleo seguro. Es alarmante observar que un $51.25 \%$ de la población con educación secundaria y media superior está ocupada, mientras que únicamente el $21.4 \%$ de educación superior, similar número de personas con los ocupados con instrucción de educación primaria en un 0.4 menos en este último. En México se coloca más fácilmente en el sector laboral una persona con menor instrucción, no así cuando se cuenta con mayor grado de conocimiento, lo que no significa obtener un ingreso suficiente para garantizar una vida digna.

\section{Gráfica 4.}

Ocupados por nivel de educación III Trimestre 2019

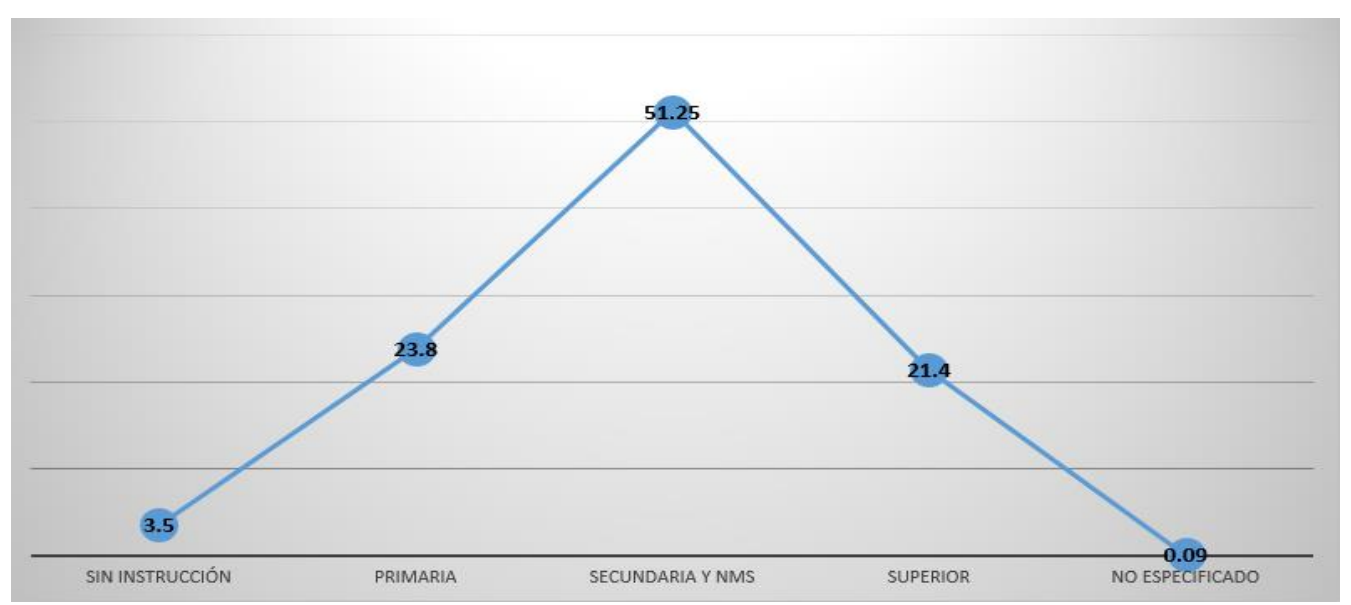

Fuente: Elaboración propia con datos obtenidos de la estadística de la STPS, Información laboral, noviembre 2019.

De acuerdo con "Education at a Glance 2018", "las expectativas laborales de aquellos con cualificaciones más bajas son menores y suelen desempeñar trabajos basados en rutinas que tienen un riesgo mayor de ser automatizados a corto y medio plazo, aumentando la probabilidad de estar desempleados". Estas disparidades en el mercado laboral pueden agravar las desigualdades sociales. En México entre más años de estudios, mayor es la posibilidad de estar en situación de "desocupado". Afirmación que se puede advertir en la gráfica 5, donde encontramos que el $20 \%$ de la 
población mexicana que cuenta con estudios de nivel medio superior se encontraba en situación de "desocupado" para el año 2019, en 10 años presento un incremento de 7\% (del 2001 al 2019), de igual manera en el mismo periodo se elevó en $14 \%$ el desempleo para personas con estudios de nivel superior, al pasar del $16 \%$ en el primer trimestre del año 2001 al $30 \%$ en el mismo trimestre, pero del 2019, contrario a ello la población "sin instrucción" disminuyó en desempleo en 3\%, los que cuentan con estudios de primaria en un $19 \%$ al pasar $34 \%$ en el primer trimestre de 2001 a $15 \%$ en el primer trimestre de 2019 .

\section{Gráfica 5.}

México: Desocupados por nivel de instrucción comparando primer semestre de los años 2001 y 2019

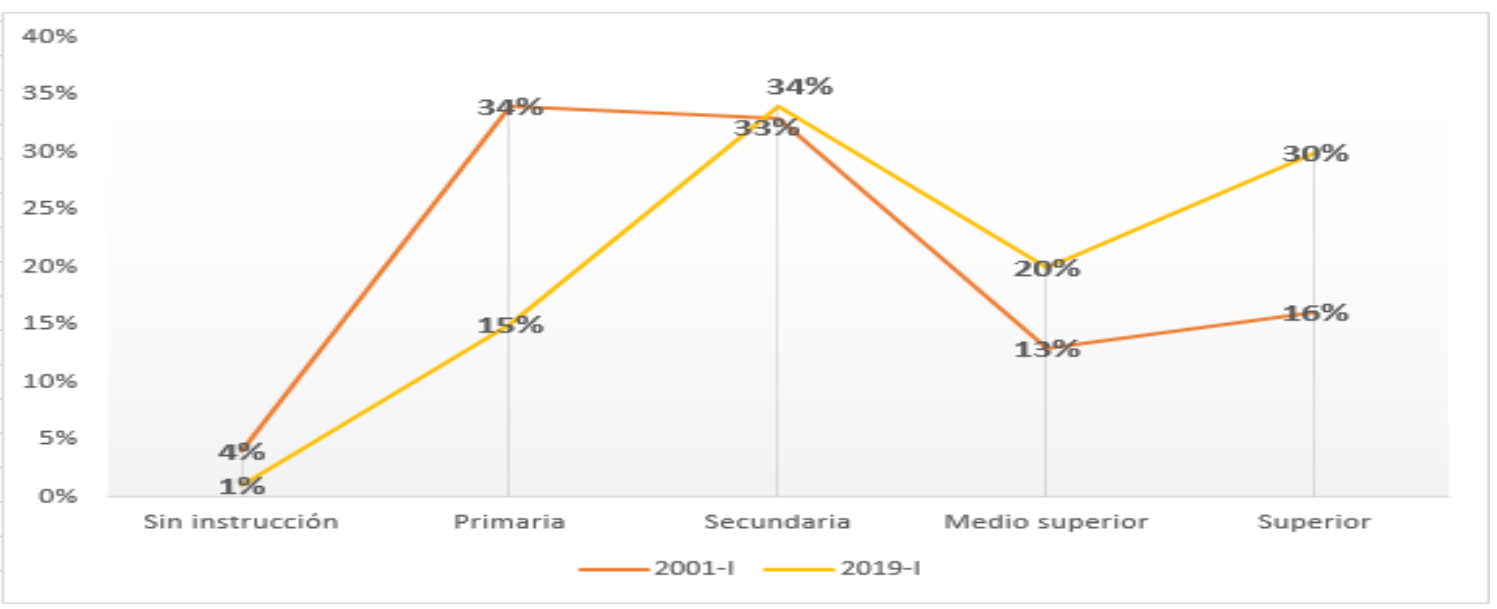

Fuente: Elaboración propia con información del Información laboral. STPS, junio 2019

Por otra parte, al hablar de productividad laboral, la OCDE (2018) señala que constituye un requisito previo fundamental para conseguir un elevado crecimiento de la producción, del empleo y de los salarios, aparte de resultar esencial para que la calidad de vida mejore a largo plazo. La misma agencia internacional indica que "las competencias son clave para mejorar la productividad laboral y los salarios, además de constituir un indicio del nivel de preparación para responder a futuros desafíos". México de acuerdo con la OCDE (2015) tiene el nivel más bajo de productividad laboral entre los países miembros, al mismo tiempo la agencia internacional indica que el limitado desempeño de México en términos de productividad puede explicarse, en parte, por el bajo nivel de competencias de su fuerza laboral. Paralelo a ello, una de las deficiencias que existen en el sistema de educación superior mexicano, se refiere a la falta de garantía en la calidad de lo que se enseña en carreras universitarias, al respecto Ramírez (2019) señala que 93.5 por ciento de las universidades privadas que hay en el país, no ofrecen carreras certificadas como "Programas de calidad", de igual forma apunta, que de acuerdo con cifras de la Secretaría de Educación Pública (SEP), sólo 145 universidades privadas en México — de las 2 mil 224 que tiene conocimiento la Asociación Nacional de Universidades e Instituciones de Educación Superior (ANUIES) - cuentan con algún programa certificado por su calidad. En contraste, las universidades públicas estatales suman el mayor porcentaje en la oferta de los llamados programas de calidad educativa, de 35 de estas escuelas superiores, 85 por ciento de sus alumnos estudia en licenciaturas garantizadas por su 
calidad. En total las 35 universidades públicas estatales ofrecen un total de mil 907 carreras que cuentan con esta certificación y que son cursadas por 960 mil 794 estudiantes en todo el país (enero, 18, 2019).

\section{CONCLUSIONES}

México tiene grandes retos que superar en torno a la Educación, elementos indispensables para garantizar el desarrollo humano y con ello poder avanzar al diseño de una cruzada educativa que forme a las nuevas generaciones. No solo hace falta replantear la forma y la cantidad de la inversión en educación, se deben tomar variables de bienestar para conocer la problemática de fondo.

La desigualdad, la pobreza y la migración descontrolada, son frenos que impiden al país poder desarrollar el potencial de sus habitantes, siendo que estos fenómenos socioeconómicos perturban el cumplimiento de los derechos humanos más esenciales, por ello el gobierno tiene que visualizar las distintas aristas, para poder destinar los recursos de manera efectiva, sin caer en paliativos mediáticos, o en tendencias que no corresponden a la realidad de la nación, no se puede seguir importando modelos que no sean coherentes con el contexto mexicano. Hay que poner de manifiesto en el centro de la discusión a la condición humana antes que a su elemento económico.

\section{FUENTES CONSULTADAS}

Banco Mundial. (s/f). Educación. https://www.bancomundial.org/es/topic/education/overview\#1 Fuentes Nieva y Barrón. (2018). Seminario CONEVAL: Desarrollo social y rendición de cuentas. https://www.coneval.org.mx/Eventos/Paginas/Seminarios-CONEVAL-Desarrollo-Social-yRendicion-de-Cuentas/Desigualdad-en-Mexico.aspx

CONEVAL. (2020). Medición de la pobreza. Índice de tendencia laboral de la pobreza al primer trimestre de 2020. https://www.coneval.org.mx/Medicion/Publishinglmages/ITLP/2020/1T2020/3\%20Porcentaje_ 1T2020.jpg

CONEVAL. (2018). Informe de Evaluación de la Política de Desarrollo Social 2018. Resumen Ejecutivo. Consejo Nacional de Evaluación de la Política de Desarrollo Social. https://www.coneval.org.mx/Medicion/Paginas/Pobrezalnicio.aspx

CONEVAL. Glosario. https://www.coneval.org.mx/Medicion/Paginas/Glosario.aspx

CEFP. (2018). Caracterización del Mercado Laboral en México. Encuesta Nacional de Ocupación y Empleo, ENOE - Primer Trimestre 2017 y 2018 -. México: Cámara de Diputados.

CEPAL. (2019). Panorama Social de América Latina. https://www.cepal.org/es/publicaciones/44969panorama-social-america-latina-2019

INEGI. (2019). Encuesta nacional de ingresos y gastos de los hogares (ENIGH) 2018. 31 de julio de 2019. 
INEGI. (2019). Resultados Encuesta Nacional de Ingresos y Gastos de los Hogares (ENIGH). 31 de julio de 2019.INEGI.

INEGI. (2019). Actualización de la medición de la economía informal, 2003-2018. Preliminar. año base 2013. Comunicado de prensa núm. 693/19 16 de diciembre de 2019 Página 1/2. https://www.inegi.org.mx/contenidos/saladeprensa/boletines/2019/StmaCntaNal/MEl2018.pdf

OCDE. (2020).¿Cómo va la vida en México?. https://www.oecd.org/statistics/Better-Life-Initiativecountry-note-Mexico-in-Spanish.pdf

OCDE. Better Life Index. http://www.oecdbetterlifeindex.org/es/topics/education-es/

OCDE. (2019). Panorama de la educación Indicadores de la OCDE 2019. https://www.educacionyfp.gob.es/dam/jcr:b8f3deec-3fda-4622-befb386a4681b299/panorama\%20de\%20la\%20educaci\%C3\%B3n\%202019.pdf

OCDE. (2019). Education at a Glase 2019. https://www.oecd.org/education/education-at-aglance/EAG2019_CN_MEX_Spanish.pdf

OCDE. (2018). La nueva Estrategia de empleo de la OCDE. Empleo de calidad para todos en un entorno laboral cambiante Situación México. https://www.oecd.org/mexico/jobs-strategyMEXICO-ES.pdf

OCDE. (2018). Education at a Glance 2018 OECD INDICATORS. https://www.oecdilibrary.org/docserver/eag-2018en.pdf?expires=1620773910\&id=id\&accname=guest\&checksum=8D31AFEC2E20328A74806 970EC65C9BC

OCDE. (2017). ¿Cómo va la vida en México?. https://www.oecd.org/statistics/Better-Life-Initiativecountry-note-Mexico-in-Espagnol.pdf

OECD. (2017). OECD Skills Strategy. Diagnóstico de la OCDE sobre la Estrategia de Competencias, Destrezas y Habilidades de México. Resumen Ejecutivo 2017.

OCDE. (2017). Estudios Económicos de la OCDE México. Enero, 2017. www.oecd.org/eco/surveys/economic-survey-méxico.htm

OCDE (2015). Serie "Mejores Políticas" México políticas prioritarias para fomentar las habilidades y conocimientos de los mexicanos para la productividad y la innovación mayo 2015. https://www.oecd.org/mexico/mexico-politicas-prioritarias-para-fomentar-las-habilidades-yconocimientos-de-los-Mexicanos.pdf

OIT. (2018). Mujeres y hombres en la economía informal: Un panorama estadístico. Tercera edición: Oficina Internacional del Trabajo, Ginebra. https://www.ilo.org/wcmsp5/groups/public/--dgreports/---dcomm/documents/publication/wcms_635149.pdf

Osorio-García, S. (2010). John Rawls: una teoría de justicia social, su pretensión de validez para una sociedad como la nuestra. Revista Relaciones Internacionales, Estrategia y Seguridad, 5(1), 137-159. https://doi.org/10.18359/ries.136

PNUD. (2020). Desarrollo Humano y COVID-19 en México: Desafíos para una recuperación sostenible. Programa de las Naciones Unidas para el Desarrollo (PNUD). https://www.mx.undp.org/content/mexico/es/home/library/poverty/desarrollo-humano-y-covid19-en-mexico-.html 
PNUD. (2020). Human Development Report 2020. The Next Frontier: Human Development and the Anthropocene. Briefing note for countries on the. http://www.hdr.undp.org/sites/all/themes/hdr_theme/country-notes/MEX.pdf

Ramírez, I. (2019). Escuelas privadas, sin filtro de calidad. Periódico El Heraldo de México. https://heraldodemexico.com.mx/pais/escuelas-privadas-sin-filtro-decalidad/?fbclid=IwAR2wWFrAX0cQH6Nn6Or1ddzpP2GY96s5aPz1cvL8w6_8q10Pmxwmm81 yiSg.

STPS. (2019). Informe Laboral, noviembre, 2019. Disponible en: http://www.stps.gob.mx/gobmx/estadisticas/pdf/perfiles/perfil\%20nacional.pdf

UNESCO. Liderar el ODS 4 - Educación 2030. https://es.unesco.org/themes/liderar-ods-4educacion-

2030\#: :text=El\%20Objetivo\%20de\%20Desarrollo\%20Sostenible,todos\%E2\%80\%9D\%20de \%20aqu\%C3\%AD\%20a\%202030. 